\title{
Pivotal Role of Non-cardiomyocytes in Electromechanical and Therapeutic Potential of Induced Pluripotent Stem Cell-Derived Engineered Cardiac Tissue
}

\author{
Hiroko Iseoka, MPharm,1,2 Shigeru Miyagawa, MD, PhD, Satsuki Fukushima, MD, PhD, Atsuhiro Saito, PhD, \\ Shigeo Masuda, MD, PhD, Shin Yajima, MD, Emiko Ito, PhD, Nagako Sougawa, PhD, \\ Maki Takeda, PhD, Akima Harada, BS, Jong-Kook Lee, MD, PhD, and Yoshiki Sawa, MD, PhD ${ }^{1}$
}

\begin{abstract}
Although engineered cardiac tissues (ECTs) derived from induced pluripotent stem cells (iPSCs) are promising for myocardial regenerative therapy, the appropriate ratio of cardiomyocytes to non-cardiomyocytes is not fully understood. Here, we determined whether ECT-cell content is a key determinant of its structure/function, thereby affecting ECT therapeutic potential for advanced heart failure. Scaffold-free ECTs containing different ratios $(25 \%, 50 \%, 70 \%$, or $90 \%)$ of iPSC-derived cardiomyocytes were generated by magnetic-activated cell sorting by using cardiac-specific markers. Notably, ECTs showed synchronized spontaneous beating when cardiomyocytes constituted $\geq 50 \%$ of total cells, with the electrical-conduction velocity increasing depending on cardiomyocyte ratio; however, ECTs containing $90 \%$ cardiomyocytes failed to form stable structures. ECTs containing $25 \%$ or $50 \%$ cardiomyocytes predominantly expressed collagen and fibronectin, whereas ECTs containing 70\% cardiomyocytes predominantly expressed laminin and exhibited the highest contractile/relaxation properties. Furthermore, transplantation of ECTs containing $50 \%$ or $70 \%$ cardiomyocytes into a rat chronic myocardial infarction model led to a more profound functional recovery as compared with controls. Notably, transplanted ECTs showed electrical synchronization with the native heart under Langendorff perfusion. Collectively, these results indicate that the quantity of non-cardiomyocytes is critical in generating functional iPSC-derived ECTs as grafts for cardiac-regeneration therapy, with ECTs containing 50-70\% cardiomyocytes exhibiting stable structures and increased cardiotherapeutic potential.
\end{abstract}

Keywords: induced pluripotent stem cells, regenerative medicine, heart failure, cell transplantation

\section{Introduction}

A DVANCED HEART FAILURE results from the loss of large numbers of viable myocytes and is a lethal disease worldwide. Although left-ventricular assist-device implantation and heart transplantation are the standard and ultimate treatments for advanced heart failure, they are critically limited by their availability, durability, and intrinsically associated complications, encouraging the development of innovative treatments, such as cardiac-regenerative therapy. Transplantation of somatic tissue-derived stem/progenitor cells, including mesenchymal stem cells or skeletal myoblasts, has been suggested for regeneration of the infarcted myocardium via cytokine-paracrine effects; however, the reported therapeutic efficacy is not dramatic and particularly limited in advanced heart failure due, in part, to insufficient numbers of myocytes remaining in the heart for functional recovery. ${ }^{3,4}$

Recently, it was suggested that the damaged myocardium can be replaced by induced pluripotent stem cells (iPSCs) or embryonic stem cell-derived engineered cardiac tissues (ECTs) containing cardiomyocytes and non-cardiomyocytes

\footnotetext{
${ }^{1}$ Department of Cardiovascular Surgery, Osaka University Graduate School of Medicine, Osaka, Japan.

${ }^{2}$ Terumo Corporation, Tokyo, Japan.

${ }^{3}$ Department of Cardiovascular Regenerative Medicine, Osaka University Graduate School of Medicine, Osaka, Japan.

(C) Hiroko Iseoka et al. 2018; Published by Mary Ann Liebert, Inc. This article is available under the Creative Commons License CC-BYNC (http://creativecommons.org/licenses/by-nc/4.0). This license permits non-commercial use, distribution and reproduction in any medium, provided the original work is properly cited. Permission only needs to be obtained for commercial use and can be done via RightsLink.
} 
to produce spontaneous regular beating. ${ }^{5,6}$ Importantly, ECT transplantation is more advantageous for graft survival, functionality, and integrity than transplantation of dissociated cardiomyocytes $^{7}$; however, in vitro protocols for ECT generation have not been optimized for clinical use in terms of phenotypic, mechanical, and electrophysiological properties. Multicellular interactions are important for enhancing the

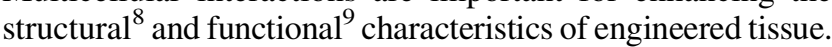
Although critical roles for non-cardiomyocytes in promoting structural stability in cardiac tissue have been suggested, ${ }^{10}$ the optimal ratio of cardiomyocytes to non-cardiomyocytes to enhance the cardiotherapeutic properties of ECTs is poorly understood. Here, we investigated whether the content of cardiomyocytes and non-cardiomyocytes in ECTs is a key determinant of ECT structure, function, and potential as an electrophysiological integration-mediated therapeutic for advanced heart failure.

\section{Materials and Methods}

\section{ECT preparation from human iPSCs}

Cardiomyogenesis was induced in human 253G1 iPSCs by using a bioreactor system as previously described. ${ }^{11}$ Cardiomyocytes were isolated from the differentiated cell preparation by using a magnetic-activated cell-sorting (MACS) system (Miltenyi Biotec, Teterow, Germany) targeting the cardiacspecific cell-surface marker CD172a. ${ }^{12}$ Separated cardiomyocytes were mixed with non-cardiomyocytes at ratios of $25 \%$, $50 \%, 70 \%$, or $90 \%$ and then plated in thermoresponsive culture dishes (Cellseed, Tokyo, Japan) to produce scaffold-free ECTs. A more detailed description of the experimental procedures is described in Supplementary Data (Supplementary Data are available online at www.liebertpub.com/tea).

\section{Flow cytometry and cell sorting}

Cultured cells were enzymatically dissociated, labeled with fluorescence-conjugated antibodies, and assessed by using the FACScantoII system (Becton Dickinson, East Rutherford, NJ). A more detailed description of experimental procedures and information of antibodies is provided in Supplementary Data.

\section{Real-time polymerase chain reaction}

Total RNA was extracted by using an RNAeasy kit (Qiagen, Hilden, Germany), and cDNA was synthesized by using a SuperScript VILO cDNA synthesis kit (Thermo Fisher Scientific, Waltham, MA, USA). Real-time polymerase chain reaction (PCR) was performed by using TaqMan PCR master mix on a Viia7 real-time PCR system (Thermo Fisher Scientific). Details on primer and probe sets can be found in Supplementary Data.

\section{Immunohistolabeling and fluorescence- intensity analysis}

ECT, dissociated single cells, or harvested hearts were fixed with $4 \%$ paraformaldehyde and labeled with primary antibodies, followed by incubation with fluorescence-conjugated secondary antibodies, counterstaining with 4',6-diamidino-2phenylindole (DAPI) or Hoechst33258, and finally analysis by confocal microscopy (Carl Zeiss, Jena, Germany). The labeled cells were captured based on their fluorescence intensity. Information of antibodies is described in Supplementary Data.

\section{Electrophysiology}

Dissociated cells were plated on $0.1 \%$ gelatin-coated plates and cultured for 5 days. Field potential, $\mathrm{Ca}^{2+}$ transient, membrane potential, and cell motion were measured. A more detailed description of the experimental procedures is provided in Supplementary Data.

\section{Epicardial ECT transplantation}

ECTs were transplanted into a chronic myocardial infarction (MI) rat model. Transthoracic echocardiography and optical mapping of transplanted whole hearts were performed. A more detailed description of the experimental procedures is provided in Supplementary Data.

\section{Results}

\section{Phenotypic characteristics of cardiomyogenically} differentiated human iPSCs

Cardiomyogenic differentiation was induced in human iPSCs to produce mixed cell preparations that included cardiomyocytes and non-cardiomyocytes. Cardiomyocytes were isolated from the cell mixture by MACS-based cardiac-specific CD172a cell surface expression. It is reported that CD172a is a specific cell-surface marker that is used for isolating iPSCsderived cardiomyocytes and is expressed in both the fetal and adult human heart. ${ }^{12}$ Notably, $92.4 \% \pm 3.0 \%$ of CD172apositive cells exhibited cardiac troponin $\mathrm{T}$ (cTnT) expression (Fig. 1A), whereas the remaining cTnT-negative cells in the total cells were positive for the fibroblast-specific marker TE-7 $(7.7 \% \pm 1.4 \%)$, vimentin $(90.3 \% \pm 4.0 \%)$, alpha-smooth muscle actin ( $\alpha$ SMA; $93.6 \% \pm 0.6 \%)$, caldesmon $(45.0 \% \pm 4.2 \%)$, calponin $(28.2 \% \pm 3.8 \%)$, CD144 $(3.3 \% \pm 0.9 \%)$, and/or the endothelial-specific marker CD31 $(4.4 \% \pm 0.4 \%)$ (Fig. 1B). Moreover, these cells barely expressed any cardiac markers, such as Nkx2.5 $(0.4 \% \pm 0.3 \%)$, $\alpha$-actinin $(1.0 \% \pm 0.9 \%)$, and cardiac myosin heavy chain (cMHC; $1.5 \% \pm 1.0 \%)$, which indicate that these cells are non-cardiomyocytes.

\section{Structures of four types of ECT associated with extracellular matrix-protein expression}

After separating into cardiomyocytes or non-cardiomyocytes, cells were mixed at ratios of $25 \%, 50 \%, 70 \%$, or $90 \%$ cardiomyocytes to $75 \%, 50 \%, 30 \%$, or $10 \%$ non-cardiomyocytes and then plated in thermoresponsive culture dishes to produce four types of scaffold-free ECTs. Thermoresponsive culture dishes enabled us to collect ECTs without artificial scaffolds, which maintained cell-cell contact as a sheet structure by lowering the culture temperature. ECTs containing $25 \%, 50 \%$, or $70 \%$ cardiomyocytes successfully formed stable structures, whereas ECTs containing 90\% cardiomyocytes unexpectedly exhibited poor structural stability (Fig. 2A). In addition, the distribution and quantity of the extracellular matrix (ECM) proteins collagen, laminin, and fibronectin in the four ECTs were examined by immunohistochemistry and real-time PCR. Interestingly, collagen I and collagen III were predominantly expressed on the surface of ECTs containing $25 \%$ or $50 \%$ cardiomyocytes, whereas ECTs containing $70 \%$ or $90 \%$ cardiomyocytes displayed 

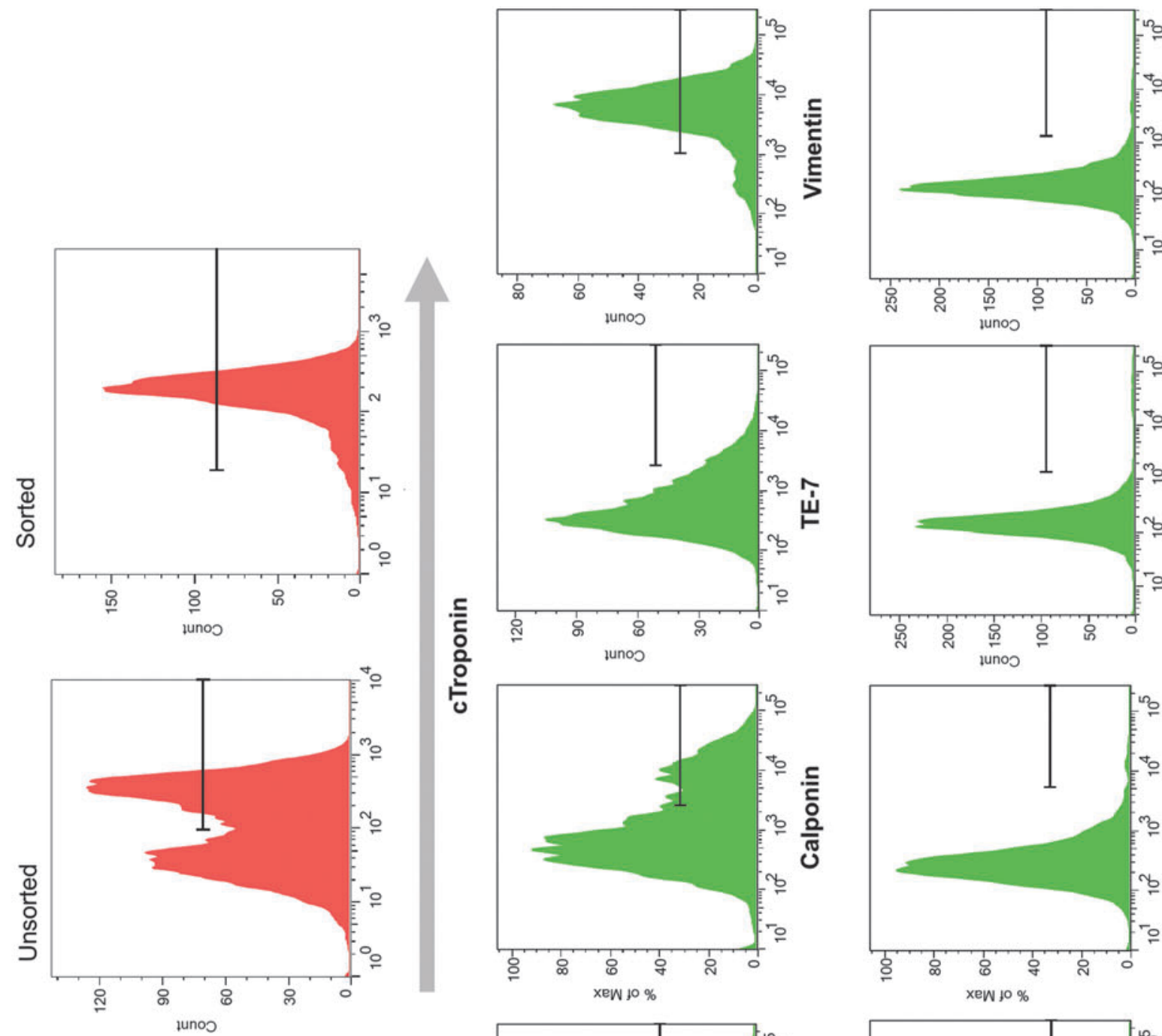

등 흥
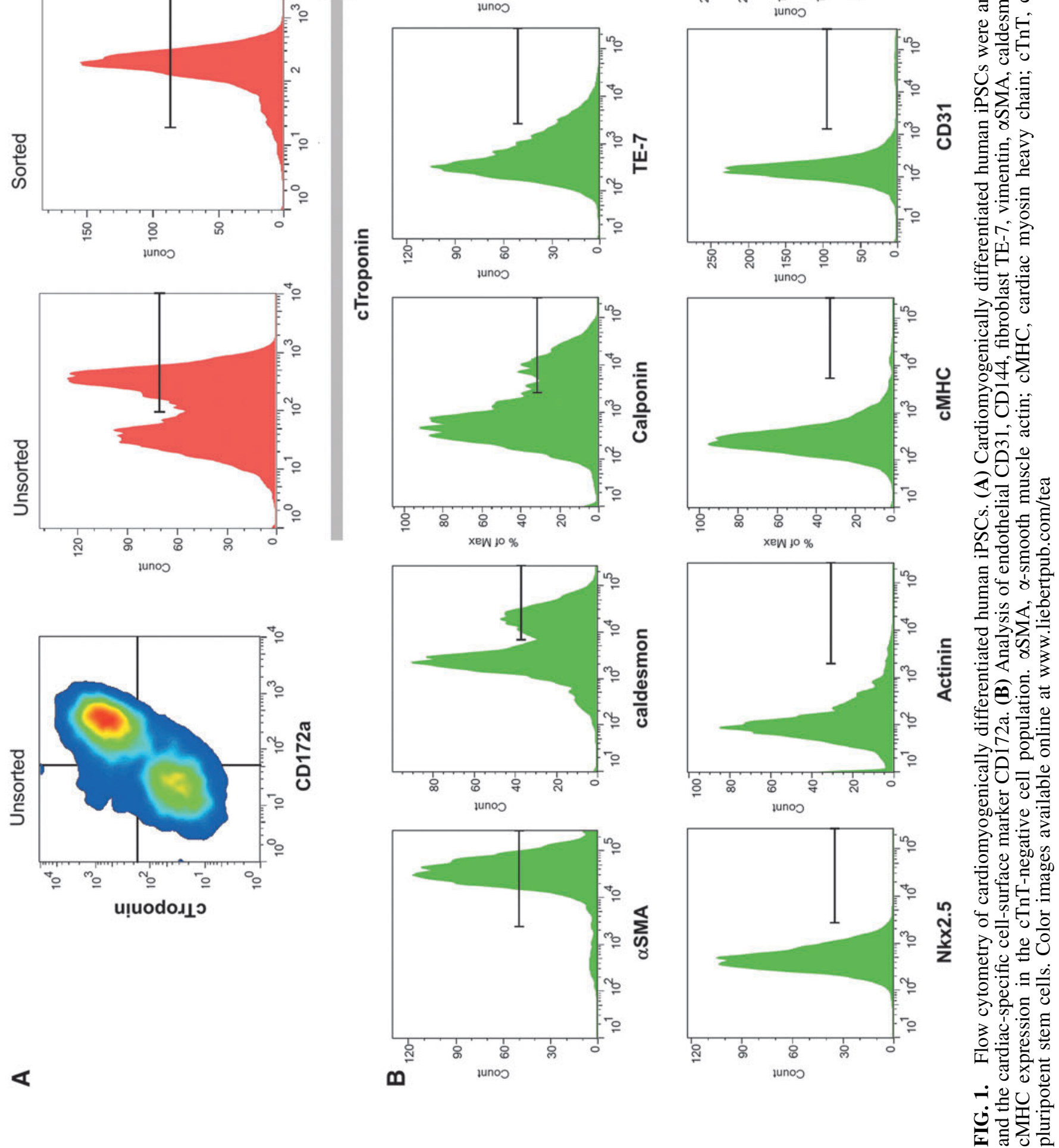

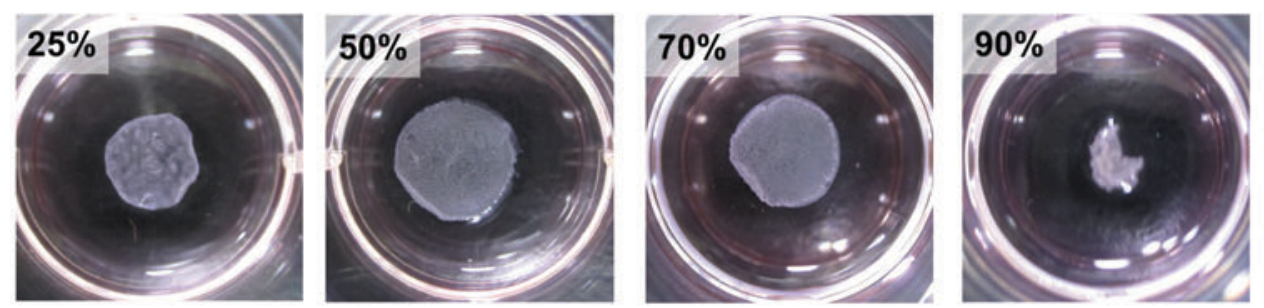

\section{B}
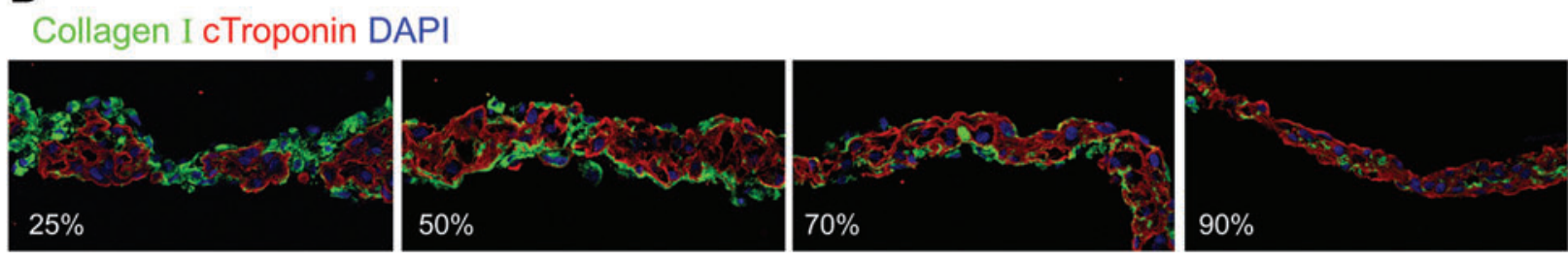

\section{Collagen III cTroponin DAPI}
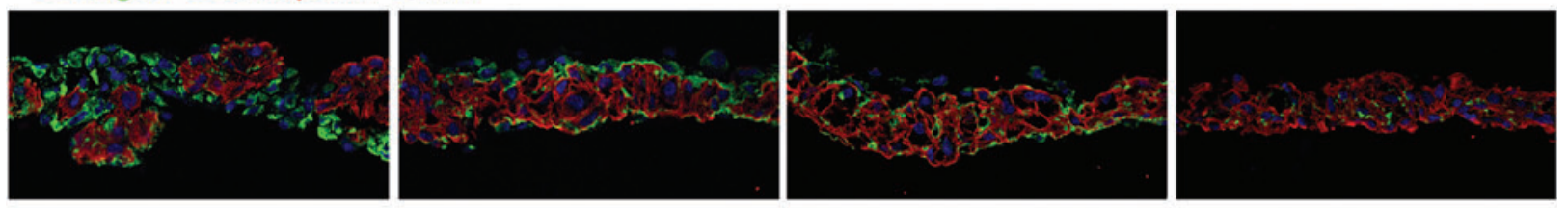

\section{Fibronection cTroponin DAPI}
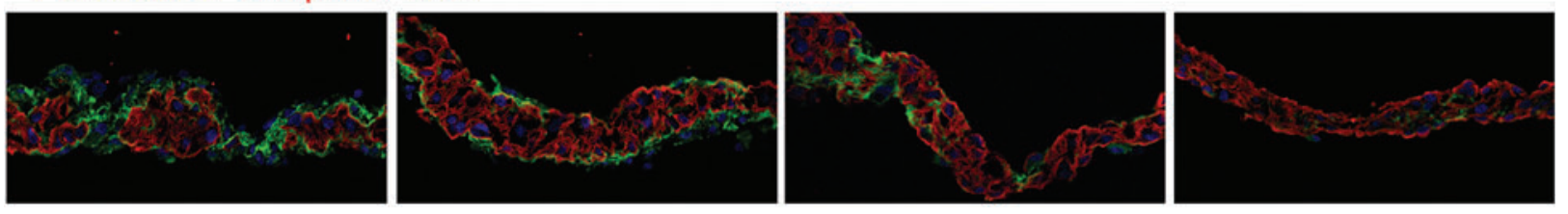

\section{Laminin cTroponin DAPI}

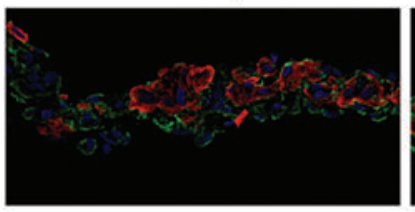

C

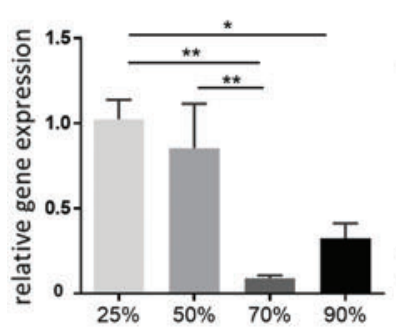

Laminin $\alpha 2$

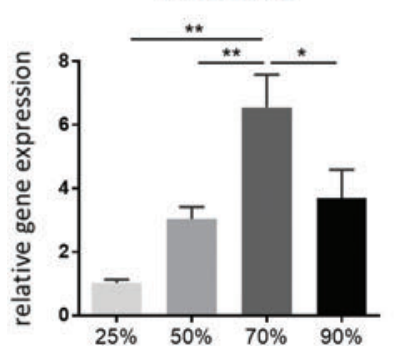

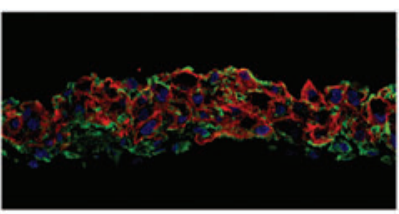

Collagen III

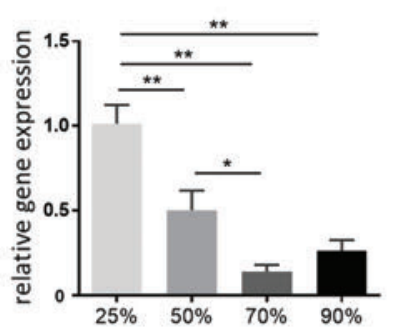

Laminin $\alpha 4$

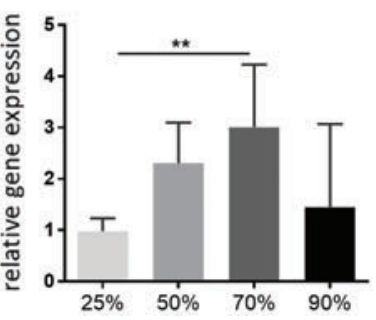

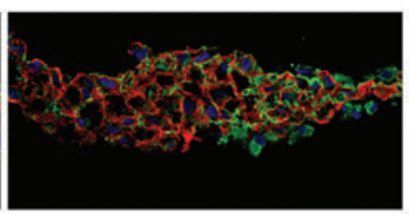

Collagen I/III

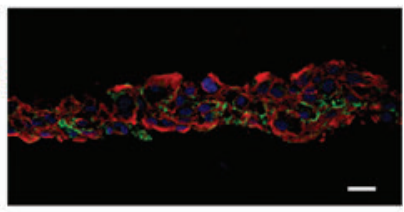

Fibronectin
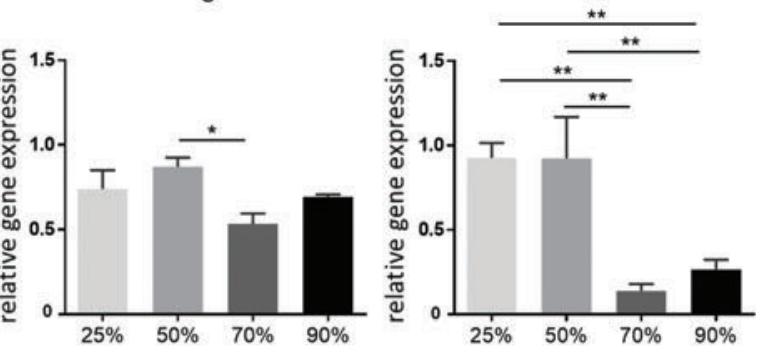

Laminin $\alpha 5$

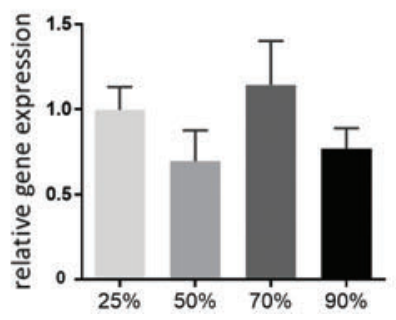

FIG. 2. Structural characteristics of ECT. (A) Scaffold-free ECTs were generated by mixing sorted cardiomyocytes and non-cardiomyocytes at different ratios $(25 \%, 50 \%, 70 \%$, or $90 \%$ cardiomyocytes to non-cardiomyocytes). Expression of the extracellular matrix proteins collagen I, collagen III, fibronectin, and laminin $\alpha 2, \alpha 4$, and $\alpha 5$ in the ECTs was assessed by (B) immunohistochemistry and (C) real-time PCR. Scale bar: $20 \mu \mathrm{m} .{ }^{*} p<0.05 ; * * p<0.01$. ECT, engineered cardiac tissues; PCR, polymerase chain reaction. Color images available online at www.liebertpub.com/tea 
minimal collagen I expression (Fig. 2B). Similarly, fibronectin was primarily expressed on the surface of ECTs containing $25 \%$ or $50 \%$ cardiomyocytes, whereas ECTs containing $70 \%$ or $90 \%$ cardiomyocytes exhibited minimal expression. In contrast, laminin was predominantly expressed in the core of ECTs containing $70 \%$ cardiomyocytes, but it was only observed sparingly in ECTs containing $25 \%$ cardiomyocytes. ECM proteins were mainly found in areas containing cTnT-negative noncardiomyocytes, suggesting that non-cardiomyocytes were the main source of ECM.

Consistently, RT-PCR analysis revealed that collagen I/ III and fibronectin expression was more pronounced in ECTs containing $25 \%$ or $50 \%$ cardiomyocytes than in ECTs containing $70 \%$ or $90 \%$ of cardiomyocytes, whereas laminin $\alpha 2$ and $\alpha 4$ expression was markedly higher in ECTs containing $70 \%$ cardiomyocytes as compared with ECTs containing $25 \%$ or $50 \%$ cardiomyocytes (Fig. 2C). Furthermore, collagen I/III ratio, a marker of stiffness and elasticity, ${ }^{14}$ was lower in ECTs containing 70\% cardiomyocytes than in counterparts containing $50 \%$ cardiomyocytes.

In addition, non-cardiomyocyte distribution was assessed by immunohistochemistry. We found that $\alpha \mathrm{SMA}-$, caldesmon-, TE-7-, vimentin-, or isolectin IB4 (IB4)-positive noncardiomyocytes localized around the cardiomyocytes (Fig. 3). cTnT-negative cells were negative for cardiac markers, such as Nkx2.5, $\alpha$-actinin, or cMHC (Fig. 3). These data indicated that cTnT-negative cells were not cardiac cells but expressed markers similar to those of myofibroblasts, which express $\alpha$ SMA and vimentin.

\section{Cardiomyocyte-dependent electrical properties of ECTs}

The electrophysiological properties of each ECT type were assessed by using a multi-electrode array system. These results showed that ECTs containing $\geq 50 \%$ cardiomyocytes produced regular, synchronized, and spontaneous beating, whereas spontaneous beating observed in ECTs containing $25 \%$ cardiomyocytes was not always synchronized (data not shown). Moreover, the electrical conduction velocity of the extracellular field potential increased depending on cardiomyocyte content (Fig. 4A, B). Furthermore, variation in interbeat interval was negatively correlated with higher cardiomyocyte ratio, although there was no significant difference in the spontaneous beating rate (Fig. 4B).

Cytoplasmic $\mathrm{Ca}^{2+}$ transients and membrane potential were also analyzed in the four ECT types by using Fluo-8 and FluoVolt, respectively. Peak ratios and rising and falling slopes associated with $\mathrm{Ca}^{2+}$ transients increased depending on content of cardiomyocytes in the ECT, whereas no significant difference was found between ECTs containing $70 \%$ or $90 \%$ cardiomyocytes (Fig. 4C, D). In addition, peak ratios and rising and falling slopes associated with membrane potential were highest in ECTs containing 70\% cardiomyocytes (Fig. 4E, F), which also exhibited the highest maximal capture pacing rate.

\section{Cardiomyocyte-dependent contractile properties of ECTS}

ECTs were examined for contractile properties by using a high-speed, camera-based, motion analysis system. Our results indicate that the contraction and relaxation velocities increased depending on cardiomyocyte content, although velocities observed in ECTs containing 90\% cardiomyocytes were lower relative to those of ECTs containing 70\% cardiomyocytes (Fig. 5A, B and Supplementary Movie S1). Consistently, contraction- and relaxation-deformation distance, which are correlated with the contractile and relaxation force of cells, ${ }^{15}$ were the highest in ECTs containing $70 \%$ cardiomyocytes. Moreover, stacked contractile- and relaxation waves demonstrated that ECTs containing 70\% cardiomyocytes exhibited the most stable waveforms (Fig. 5C).

In addition, the distribution and quantity of contractile proteins such as myosin light chain (MLC)2a and MLC2v in cardiomyocytes for each ECT were assessed by immunohistochemistry. We observed that most cTnT-positive cells were also positive for MLC2v, but rarely expressed MLC2a in all four ECT types (Fig. 6A). Interestingly, the ratio of MLC2v single-positive cells increased depending on cardiomyocyte percentage, whereas no significant difference was found in the proportion of MLC2a single-positive cells among the groups. Moreover, the relative presence of MLC2 $v$ and MLC2a double-positive cells was negatively correlated with cardiomyocyte content and, consequently, that in ECTs containing 90\% cardiomyocytes was the lowest (Fig. 6B).

Next, we performed real-time PCR analysis to examine the expression of contractile proteins (MLC2a and $M L C 2 v)$, ion-channel/ $\mathrm{Ca}^{2+}$-handling proteins (sarcoplasmic reticulum $\mathrm{Ca}^{2+}$ ATPase 2A [SERCA2A], cardiac calsequestrin $[C A S Q]$, and ryanodine receptor 2 [RYR2]), and the gapjunction protein connexin $43\left(\right.$ GJA1) in $\mathrm{CD} 172 \mathrm{a}^{+}$cardiomyocytes in the four ECT types. Expression of $M L C 2 v$ and $M L C 2 a$ increased depending on cardiomyocyte content (Fig. 6C). In addition, SERCA2A and RYR2 expression was significantly higher in ECTs containing 70\% cardiomyocytes than in those containing $25 \%$ or $50 \%$ cardiomyocytes, whereas connexin 43 expression was not significantly different among the four types, which was subsequently confirmed by immunohistochemistry (Fig. 6D).

We then examined the production of cytokines/chemokines in the ECTs, including vascular endothelial growth factor (VEGF), stem cell factor (SCF), and stromal cell-derived factor-1 (SDF-1) (Fig. 6E). Notably, VEGF and SCF expression was significantly higher in ECTs containing $50 \%$ or $70 \%$ cardiomyocytes than in counterparts containing $25 \%$ or $90 \%$ cardiomyocytes, whereas SDF-1 expression was significantly higher in ECTs containing 25\%, 50\%, and 70\% cardiomyocytes than in those containing $90 \%$ cardiomyocytes.

\section{Therapeutic potential of ECTs in a chronic MI rat model}

To assess ECT therapeutic potential, grafts containing $25 \%, 50 \%$, or $70 \%$ cardiomyocytes were transplanted over the epicardial surface of chronic MI rat hearts, and cardiac function was evaluated by echocardiography before and at 2 and 4 weeks after ECT transplantation. Because of the absence of ECM, the cell mixture containing 90\% cardiomyocytes failed to provide sufficient cell-cell contact and detached as aggregates or single cells, resulting in an inability to form a stable structure. Because the ECT containing $90 \%$ cardiomyocytes did not have a structure that could be applied to transplantation, we could not test it in vivo. Significantly, we observed that transplantation of ECT containing $70 \%$ and $50 \%$ cardiomyocyte resulted in substantial improvement of 
aSMA cTroponin DAPI
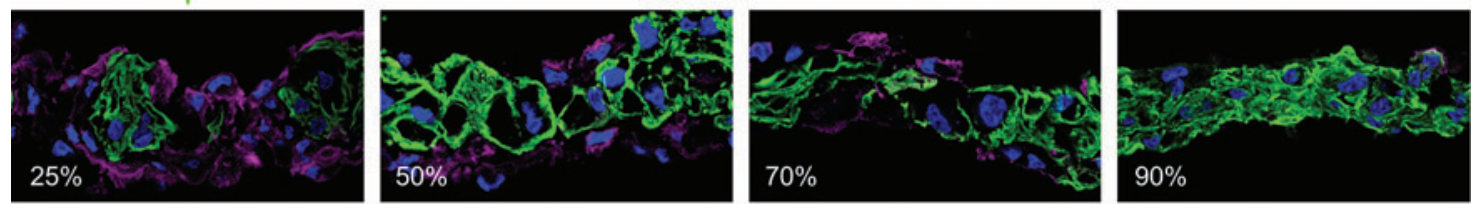

caldesmon cTroponin DAPI
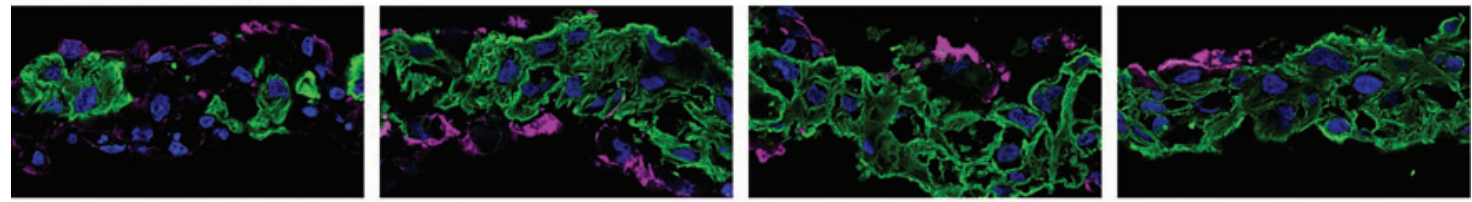

TE-7 cTroponin DAPI
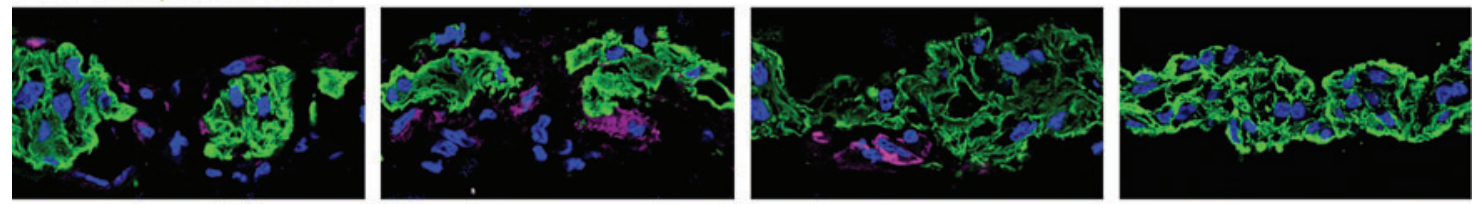

vimentin cTroponin DAPI
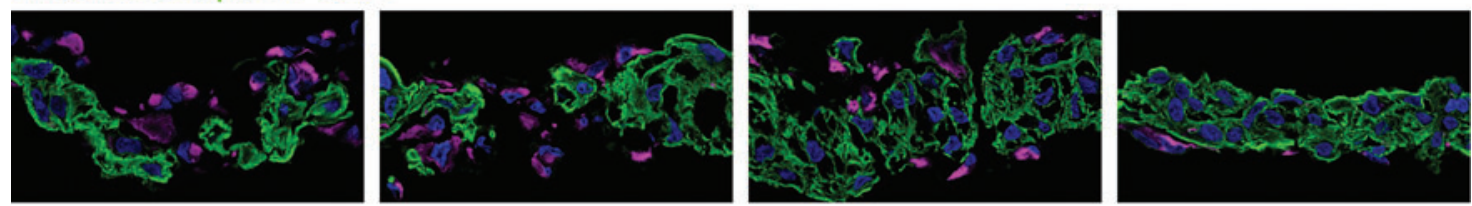

IB4 cTroponin DAPI
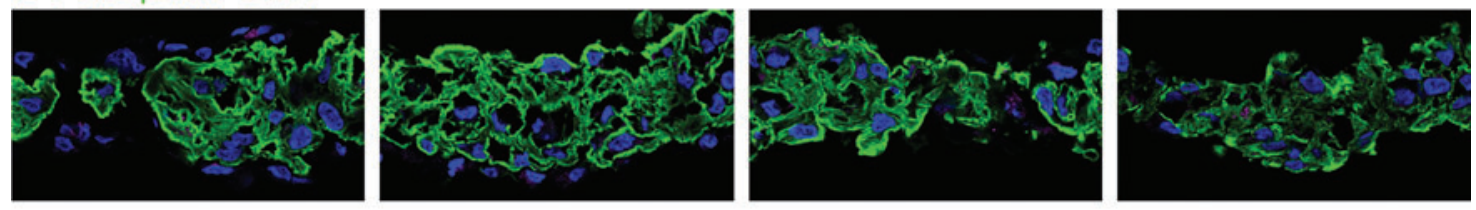

Nkx2.5 cTroponin DAPI
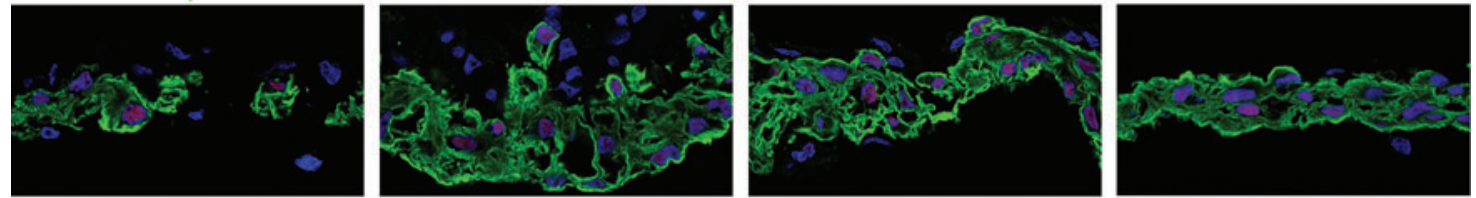

$\alpha$-actinin cTroponin DAPI
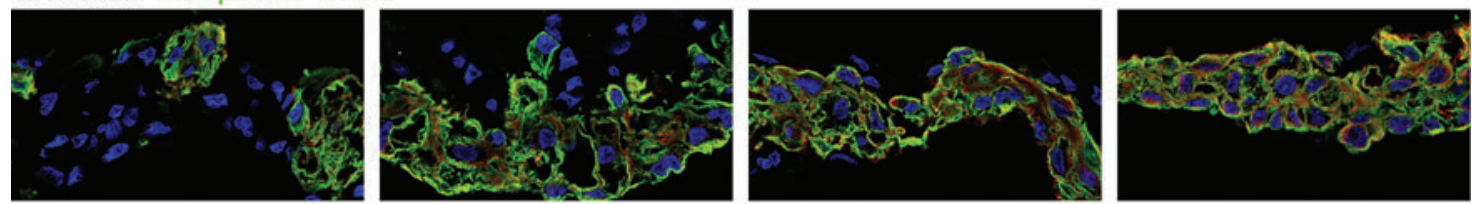

\section{cMHC cTroponin DAPI}
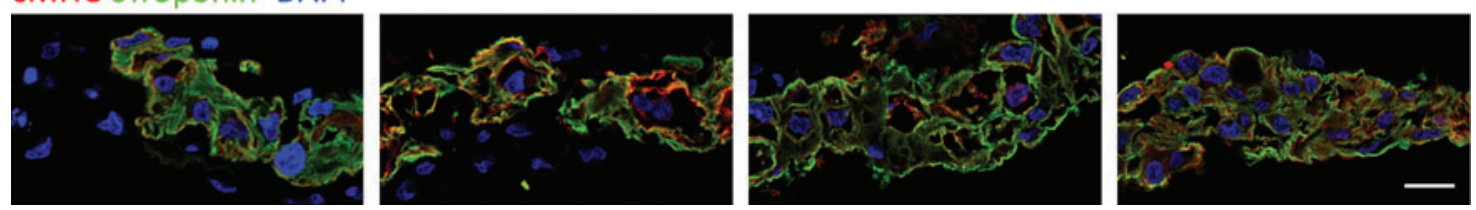

FIG. 3. Distribution of non-cardiomyocytes was examined by immunohistochemistry. Scale bar: $20 \mu \mathrm{m}$. IB-4, isolectin B4. Color images available online at www.liebertpub.com/tea 
A

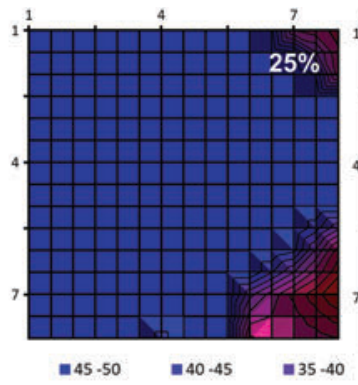

B
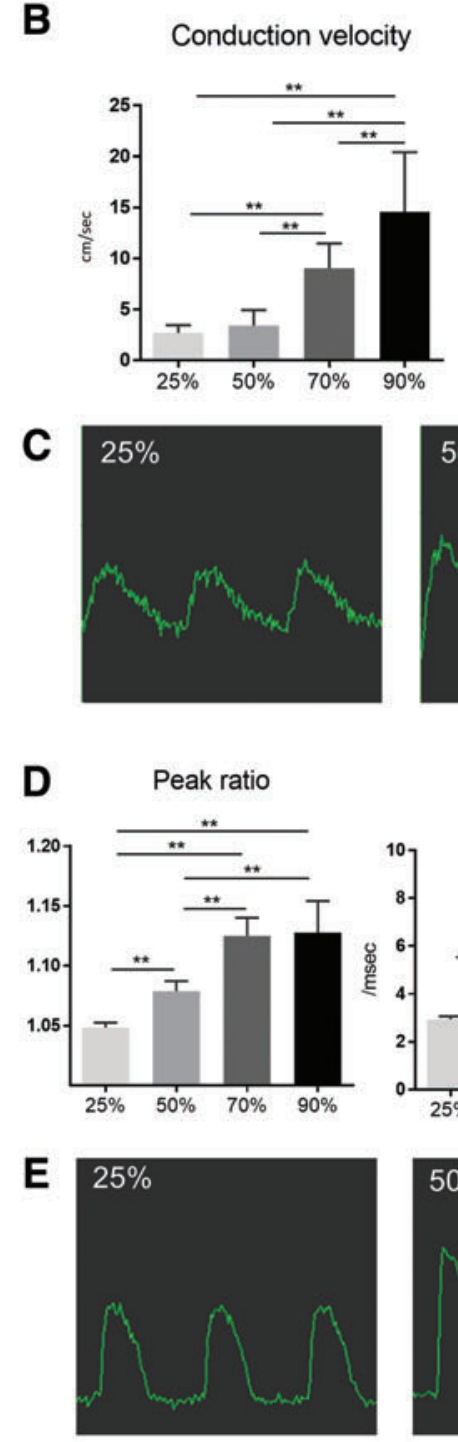

$\mathbf{F}$

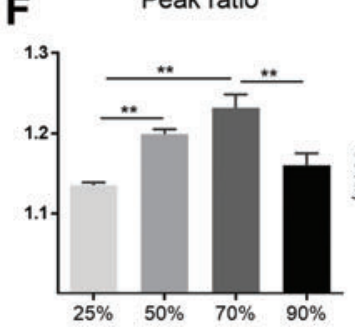

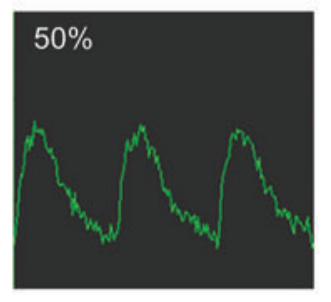
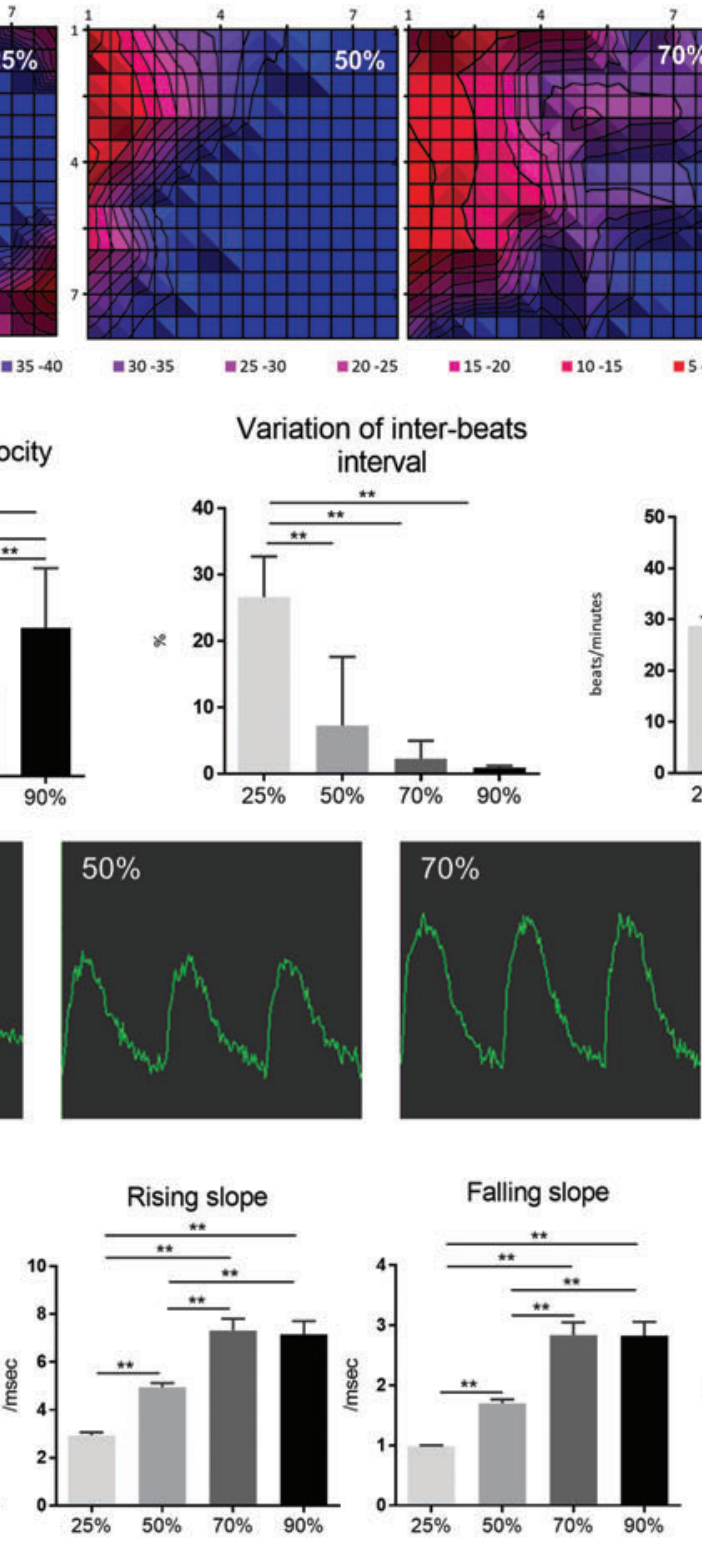

Variation of inter-beats
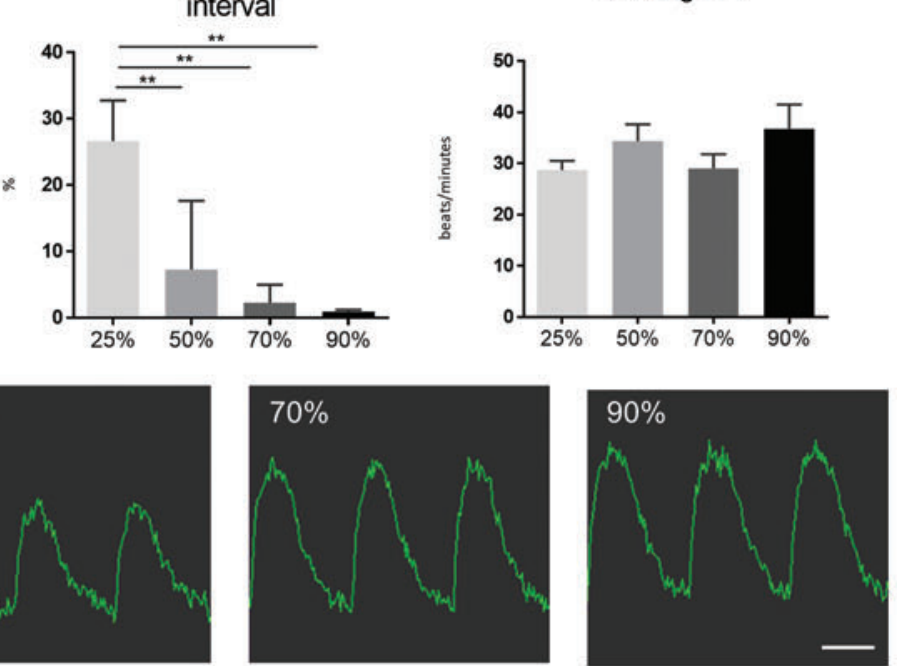

Maximally captured pacing rate
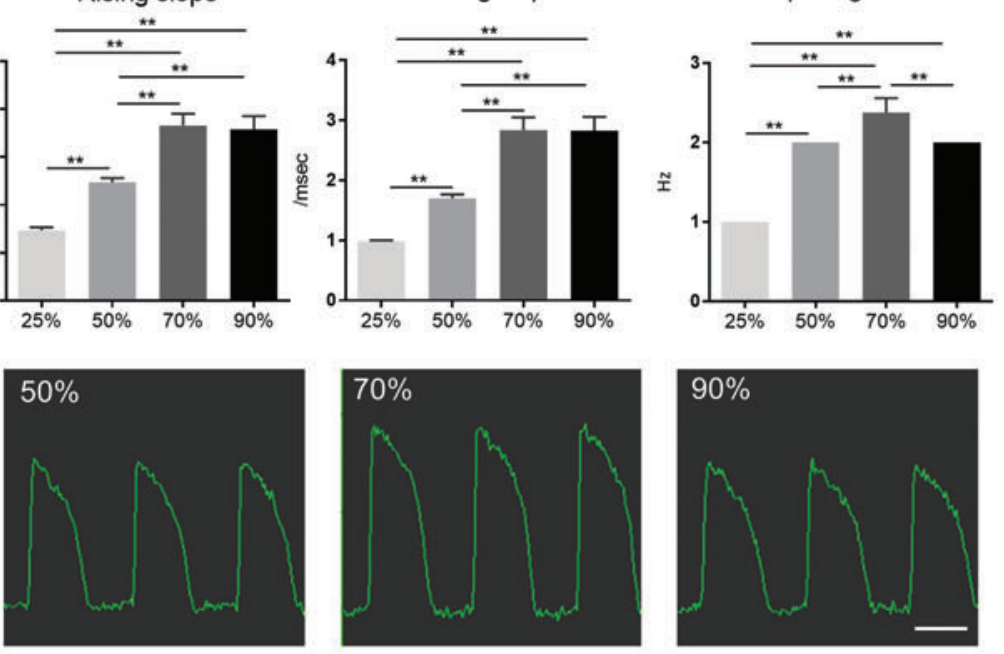

Maximally captured pacing rate
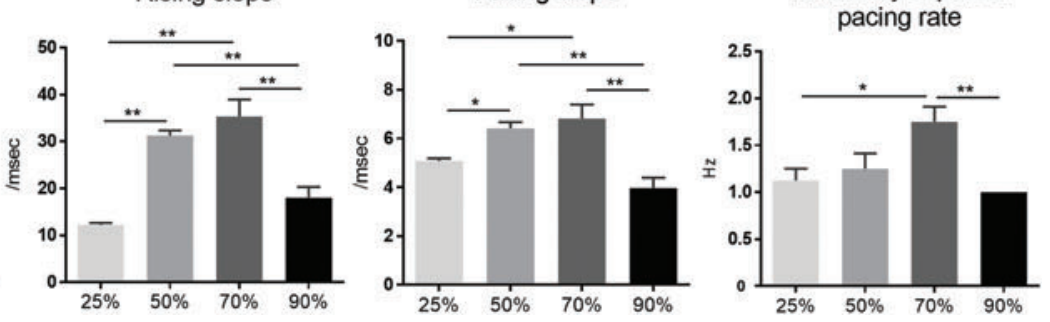

FIG. 4. Electrophysiological properties of ECTs. (A) Representative propagation map of field potential generated by the multi-electrode-array system. (B) Conduction velocity of field potential and variations in interbeat-interval and beating rate. (C) Representative wave forms associated with $\mathrm{Ca}^{2+}$ transients and (E) representative wave forms associated with membrane potential under a pacing rate of $1 \mathrm{~Hz}$. Peak ratios, rising/falling slopes, and maximal-pacing rate of (D) $\mathrm{Ca}^{2+-}$ transient and $(\mathbf{F})$ membrane potential were analyzed by the functional drug-screening system/ $\mu$ CELL system. Scale bar: $1 \mathrm{~s}$. ${ }^{*} p<0.05 ;{ }^{*} p<0.01$. BPM, beats per minute. Color images available online at www.liebertpub.com/tea 


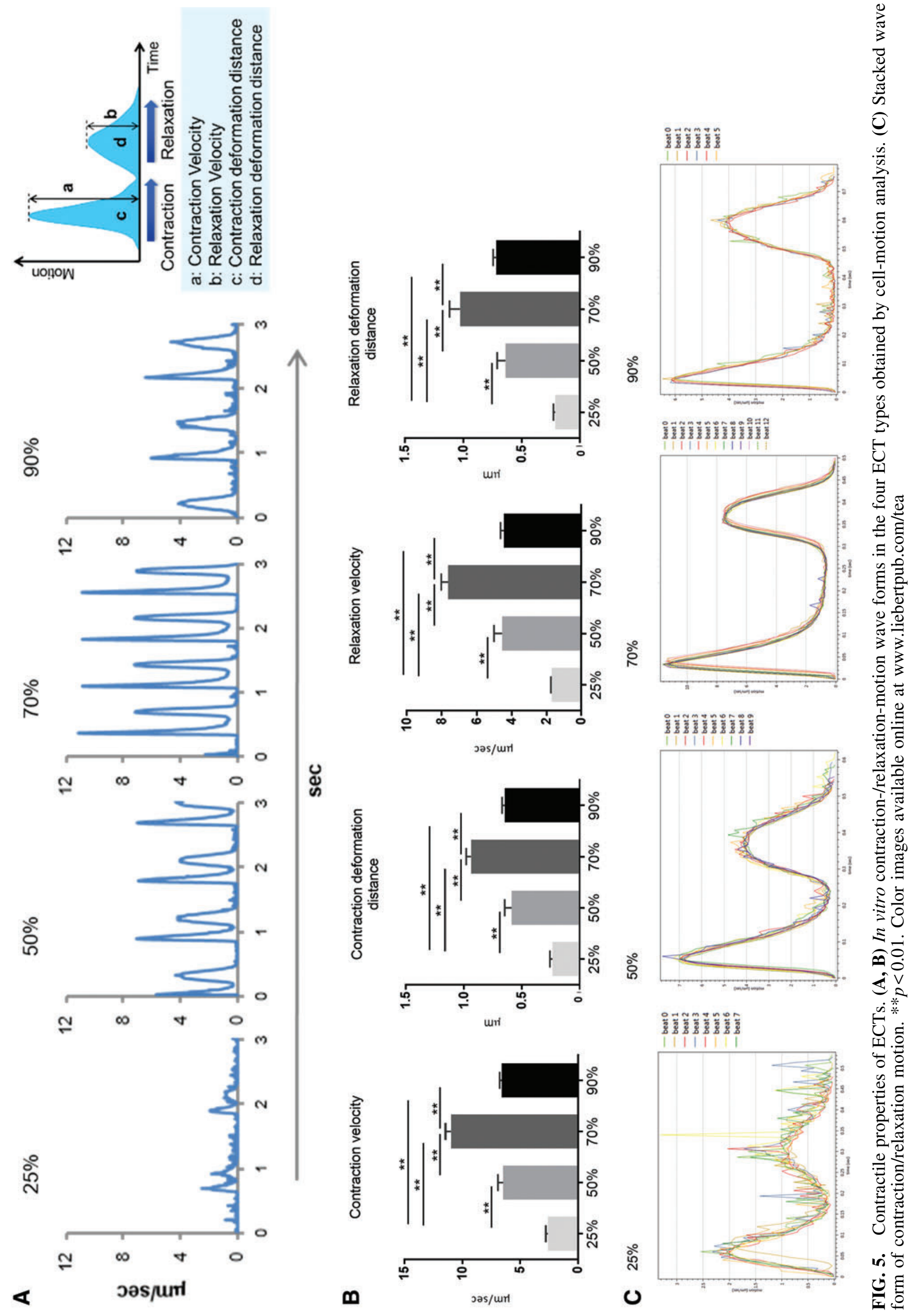




\section{A}

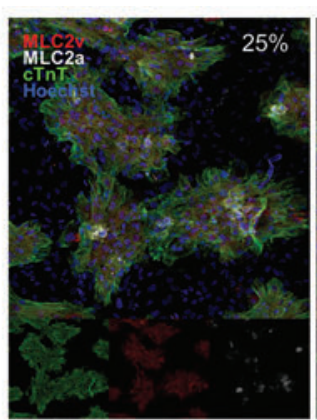

B

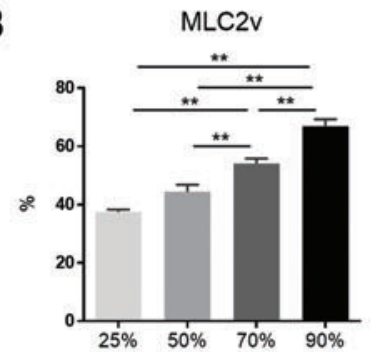

C
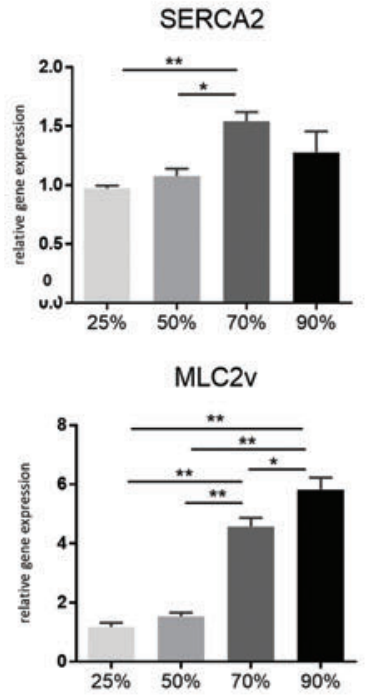
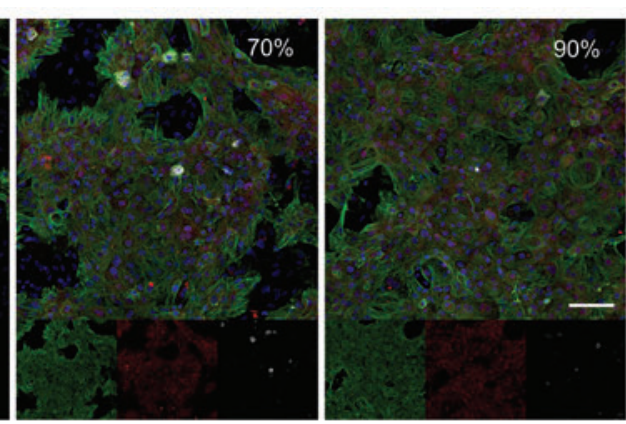

MLC2a

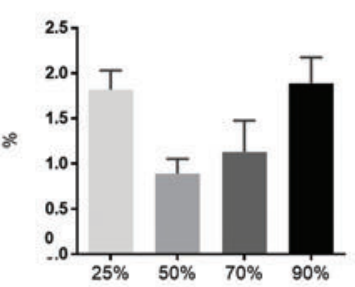

Calsequestrin 2

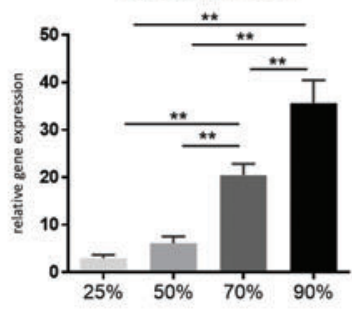

MLC2a
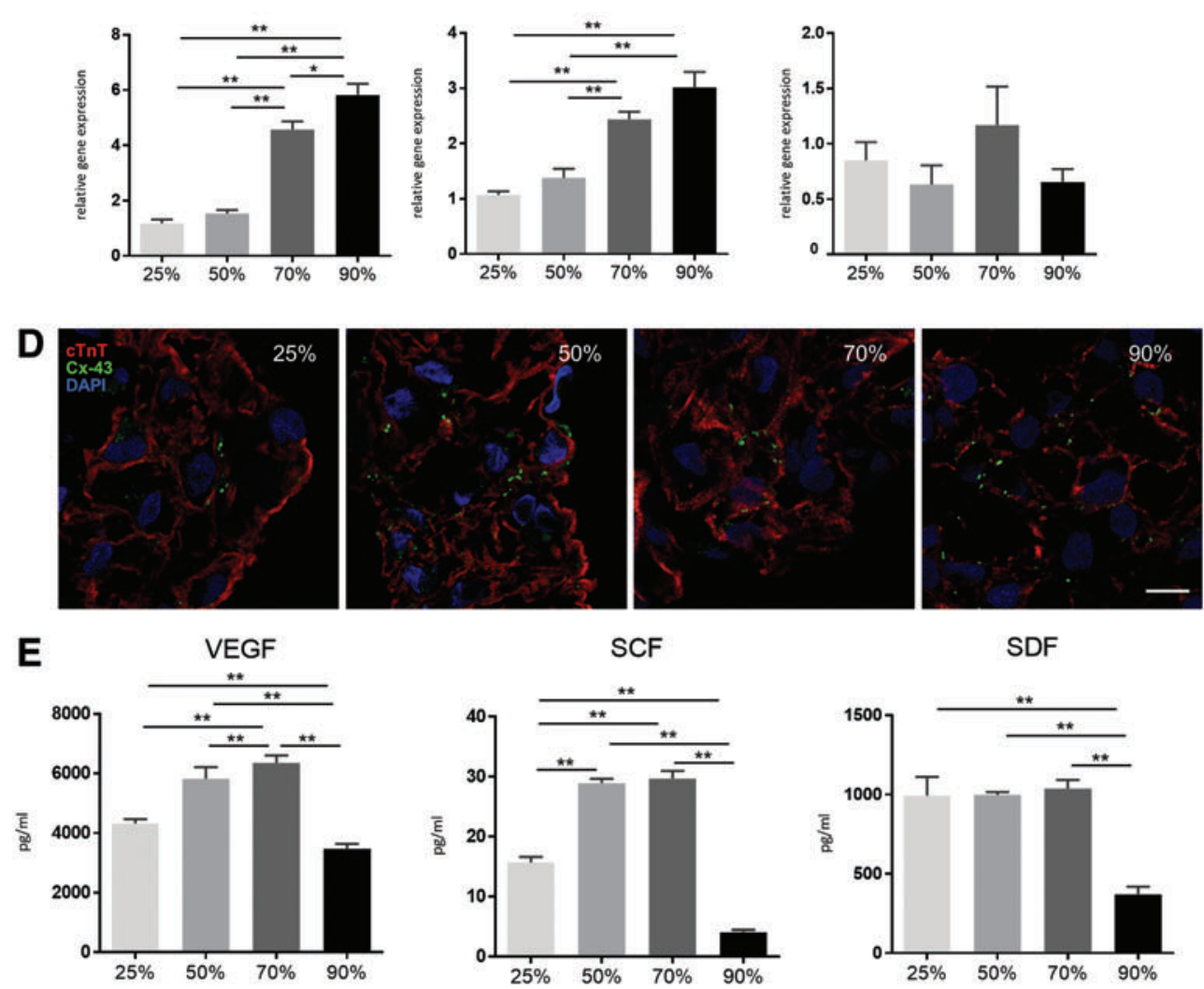
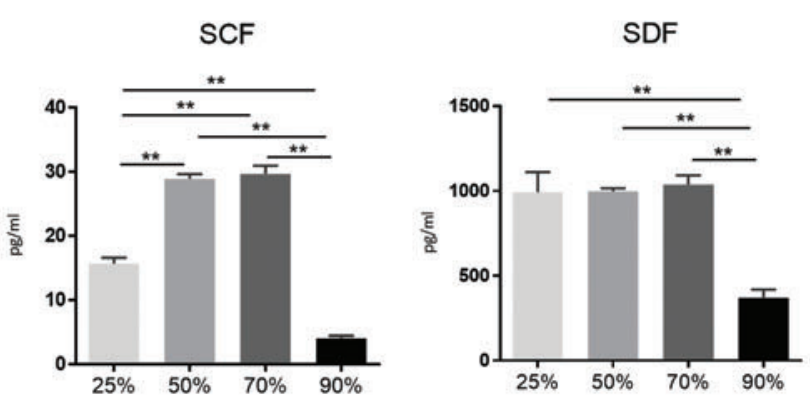

FIG. 6. Immunohistochemistry and real-time PCR analysis in the four ECT types. (A) MLC2v and MLC2a expression in cTnT-positive cells of the four ECT types was assessed by immunohistochemistry, followed by (B) quantitative analysis using a high-content imaging system (scale bar: $100 \mu \mathrm{m}$ ). (C) SERCA2A, CASQ, RYR2, MLC2v, MLC2a, and connexin 43 (GJAl) expression in the four ECT types was assessed by realtime PCR. (D) Connexin 43 localization was assessed by immunohistochemistry (scale bar: $10 \mu \mathrm{m}$ ). (E) In vitro cytokine/chemokine production in ECTs was determined by fluorescencedyed microsphere-based immunoassay. $* p<0.05$; $* * p<0.01$. CASQ, cardiac calsequestrin; MLC, myosin light chain; RYR2, ryanodine receptor 2; SERCA2A, sarcoplasmic reticulum $\mathrm{Ca}^{2+}$ ATPase 2A. VEGF, vascular endothelial growth factor; SCF, stem cell factor; SDF, stromal cell-derived factor. Color images available online at www.liebertpub .com/tea 
A
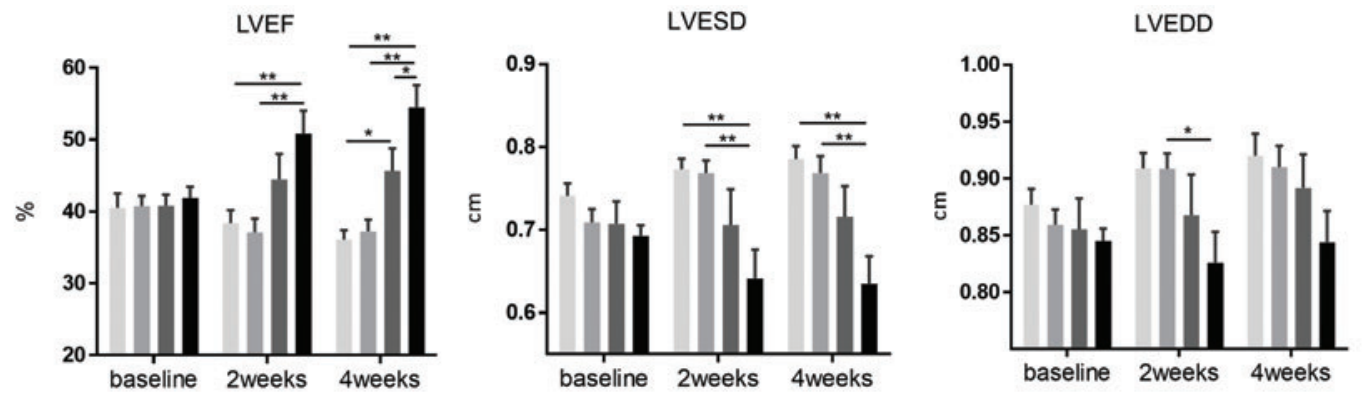

$$
\begin{aligned}
& \text { control } \\
& 25 \% \\
& 50 \% \\
& 70 \%
\end{aligned}
$$
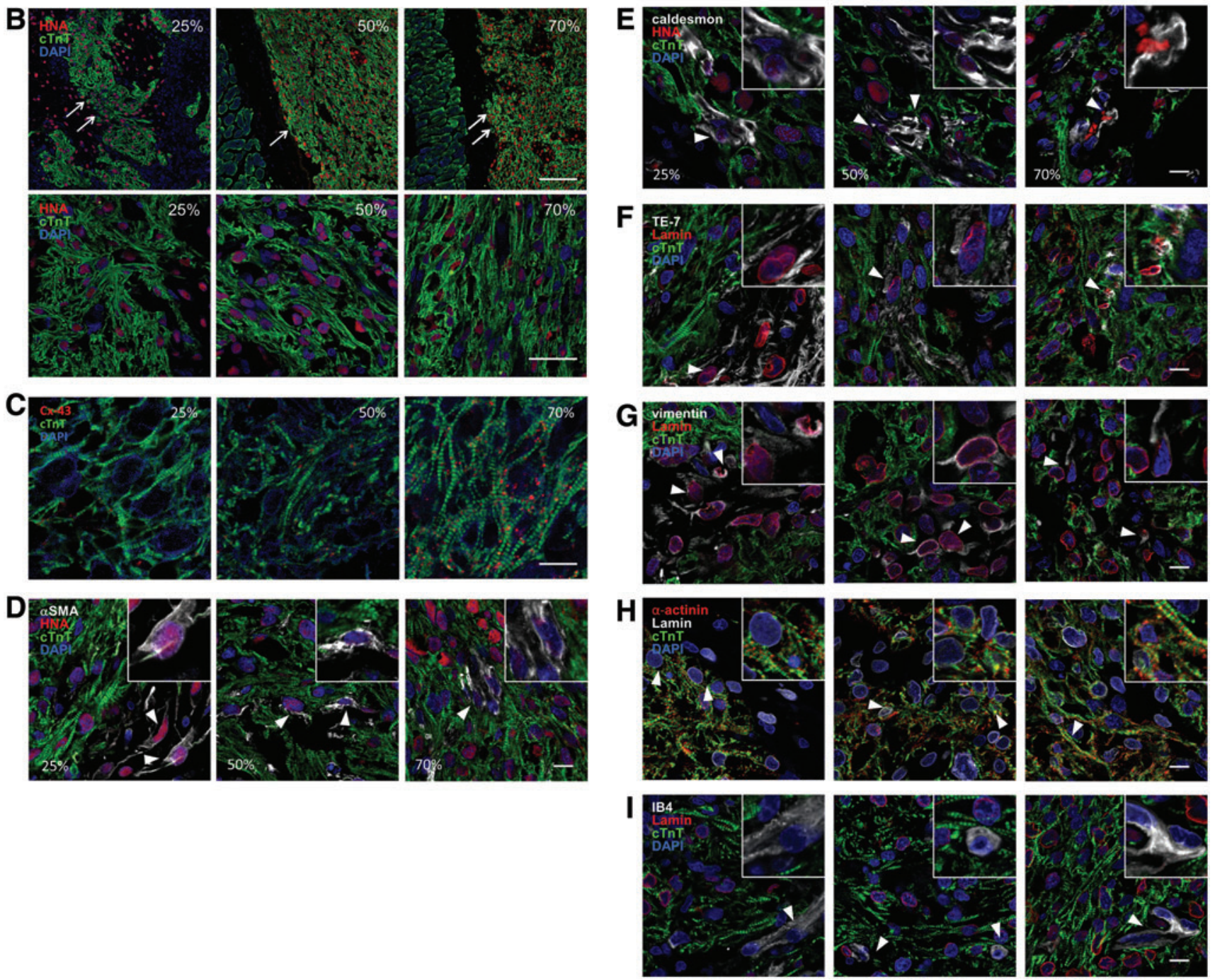

FIG. 7. In vivo analysis of ECTs transplanted into a rat model of MI. Three ECT types $(25 \%, 50 \%$, or $70 \%$ cardiomyocytes) were transplanted by left thoracotomy into nude rats subjected to left coronary artery ligation 2 weeks before transplantation. Sham rats served as controls. (A) LVEF, LVESD, and LVEDD were serially assessed by transthoracic echocardiography, whereas distribution and survival of the grafted ECT (arrows) at 4 weeks were assessed by (B-I) immunohistochemistry for cTnT, HNA, connexin 43, aSMA, caldesmon, TE-7, vimentin, $\alpha$-actinin, IB4, and human-specific lamin A/C. Inset images show magnified views. Arrow heads; (D) HNA-positive/aSMA-positive cells, (E) HNA-positive/caldesmon-positive cells, (F) Lamin-positive/TE-7-positive cells, (G) Lamin-positive/vimentin-positive cells, (H) Lamin-positive/ $\alpha$-actinin-positive cells, (I) Lamin-negative/IB4-positive cells. Scale bars: $50 \mu \mathrm{m}$ (B, upper panels), $20 \mu \mathrm{m}$ (B, bottom panels), and $10 \mu \mathrm{m}(\mathbf{C}-\mathbf{I})$. $* p<0.05 ; * * p<0.01$. HNA, human nuclear antigen; LVEDD, left-ventricular end-diastolic dimensions; LVEF, left-ventricular ejection fraction; LVESD, left-ventricular end-systolic dimensions; MI, myocardial infarction. Color images available online at www.liebertpub.com/tea 
left-ventricular ejection fraction (LVEF) at 2 and 4 weeks post-transplantation, which gradually decreased in the control group over the 4-week period (Fig. 7A and Supplementary Fig. S1). Consequently, LVEF was highest in the $70 \%$ cardiomyocyte group at 4 weeks, whereas the $50 \%$ cardiomyocyte group exhibited a significantly higher LVEF relative to the $25 \%$ cardiomyocyte and control groups. Moreover, left-ventricular end-systolic dimensions were significantly decreased in the $70 \%$ cardiomyocyte group as compared with that in the control and $25 \%$ cardiomyocyte groups at 4 weeks after transplantation, whereas no significant difference was observed in left-ventricular end-diastolic dimensions (Fig. 7A).

Furthermore, the structure and distribution of the transplanted ECTs after 4 weeks were assessed by immunohistochemistry by using antibodies for human nuclei-specific marker human nuclear antigen (HNA) and human-specific lamin A/C. Transplanted ECTs remained over the surface of native myocardia in all groups, whereas distribution, number, and structural characteristics of cTnT- and HNA-doublepositive cells (indicating grafted cardiomyocytes) were substantially different among the groups (Fig 7B). cTnT- and HNA-positive cells were surrounded by cTnT-negative/ HNA-positive grafted non-cardiomyocytes in the $25 \%$ cardiomyocyte group, whereas cTnT- and HNA-positive cells were in contact with HNA-negative native host tissue in the $70 \%$ cardiomyocyte group. Interestingly, cTnT- and HNA-positive cells were denser in grafts of the $70 \%$ cardiomyocyte group than in those of the $25 \%$ cardiomyocyte group. Moreover, cTnT- and HNA-positive cells displayed more organized intercellular alignment and mature sarcomere structure in the $70 \%$ cardiomyocyte group relative to that in the $25 \%$ cardiomyocyte graft. Furthermore, connexin 43 expression was markedly higher in $70 \%$ cardiomyocyte grafts than in all groups (Fig. 7C). In addition, cTnT-negative/ HNA-positive or human-specific lamin A/C-positive, grafted non-cardiomyocytes showed a relatively higher expression of $\alpha$ SMA or vimentin than TE-7 or caldesmon, and little expression of $\alpha$-actinin, consistent with the expression observed in our in vitro analysis (Fig. 7D-H). IB4-positive cells were negative for human-specific lamin A/C, suggesting that these cells were derived from the host tissue (Fig. 7I).

Most significantly, recipients of the $50 \%$ or $70 \%$ cardiomyocyte grafts showed significant attenuation in myocardial fibrosis and increased capillary density as compared with the control and 25\% cardiomyocyte groups (Fig. 8A-C). Consistently, RT-PCR analysis of the cardiotherapeutic cytokines $V E G F, S D F-1$, and hepatocyte growth factor $(H G F)$ in the peri-infarct area of the native myocardium revealed that $H G F$ expression was significantly higher in the $50 \%$ and $70 \%$ cardiomyocyte groups than in the control, whereas $S D F-1$ expression was significantly greater in the 50\% cardiomyocyte group (Fig. 8D).

\section{Electrical coupling between transplanted ECT and native myocardium}

Electrical coupling between the transplanted ECT and native myocardium was analyzed by epicardial optical mapping of Langendorff-perfused hearts. For this, the fluorescent $\mathrm{Ca}^{2+}$ indicator GCAMP2 was expressed in the transplanted ECT, which enabled the dynamic visualiza- tion of transplanted ECT-specific $\mathrm{Ca}^{2+}$ transients over the native myocardium. Hence, ECTs containing $50 \%$ or $70 \%$ cardiomyocytes, but not those containing $25 \%$ cardiomyocytes, produced $\mathrm{Ca}^{2+}$ transients that synchronized with membrane potential, as well as native cardiac activity as assessed by electrocardiography (Fig. 8E and Supplementary Movie S2).

\section{Discussion}

This study reports the different phenotypic, structural, and electrophysiological properties of human iPSC-derived ECTs based on their therapeutic post-transplantation effects exhibited in chronic MI rat hearts. These effects were dependent on ECT content, specifically the ratio of cardiomyocytes to non-cardiomyocytes. Notably, ECTs containing $25 \%$ cardiomyocytes predominantly expressed collagen and fibronectin, whereas ECTs containing 70\% cardiomyocytes predominantly expressed laminins. ECTs containing 25\% and $70 \%$ cardiomyocytes displayed stable structures as scaffold-free engineered tissue; however, those containing 90\% cardiomyocytes surprisingly displayed an unstable structure in vitro. Electrophysiologically, ECTs containing $70 \%$ cardiomyocytes displayed a faster conduction velocity with less interbeat-interval variation, higher peak ratios of $\mathrm{Ca}^{2+}$ transients and membrane potential, and higher maximalcaptured pacing rates as compared with ECTs containing $25 \%$ cardiomyocytes. Similarly, ECTs containing $70 \%$ cardiomyocytes showed a mechanical advantage with greater contraction/relaxation velocities and increased MLC2v expression compared with those containing 25\% cardiomyocytes. Furthermore, the $70 \%$ cardiomyocyte grafts resulted in a more profound functional recovery, with better alignment and increased HGF expression in chronic MI hearts of nude rats. Importantly, electrophysiological coupling between the native heart and the engrafted ECT was intact in those containing $70 \%$ cardiomyocytes, but not in the grafts containing $25 \%$ cardiomyocytes.

This study demonstrated that the structure of scaffold-free ECTs was determined by the presence of non-cardiomyocytes, which played pivotal roles in ECM production. Noncardiomyocytes mainly expressed markers similar to those of myofibroblasts.

Interestingly, the relative presence of non-cardiomyocytes appeared to partially determine the expression of ECM proteins, including collagen I/III, fibronectin, or laminins, leading to different electrophysiological, mechanical, and cardiotherapeutic properties in the ECTs. Accumulated collagen or fibronectin between cardiomyocytes in ECTs containing 25\% cardiomyocytes might inhibit ECT electrical propagation and contractile potentials in vitro. In addition, this group of ECTs exhibited fewer functional cardiomyocytes than those containing $50 \%$ or $70 \%$ cardiomyocytes. Collagen I provides rigidity, whereas collagen III promotes elasticity of cardiac tissue ${ }^{16}$; thus, the lower collagen I/III ratios in ECTs containing 70\% cardiomyocytes are indicative of increased ECT flexibility and enhanced contractility. Moreover, ECM composition changes with developmental age. ${ }^{17}$ Fibronectin is abundant in fetal and neonatal hearts, whereas laminin and collagen are more pronounced in adults. Therefore, the increased laminin expression in ECTs containing $70 \%$ cardiomyocytes may reflect maturation 
A
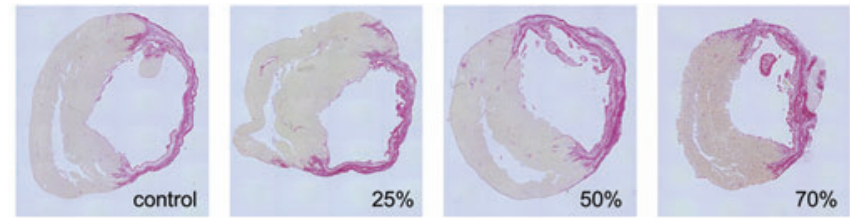

B
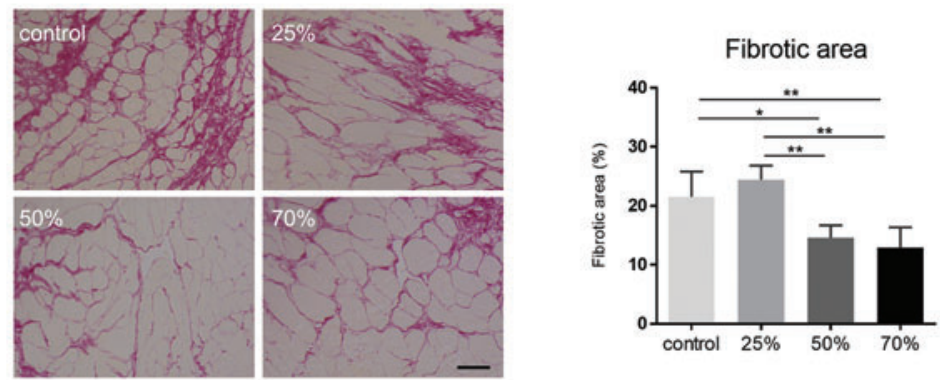

C
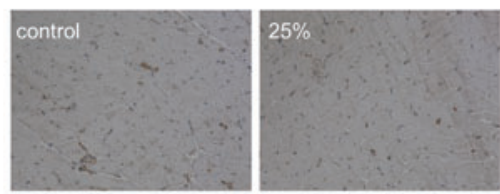

$50 \%$
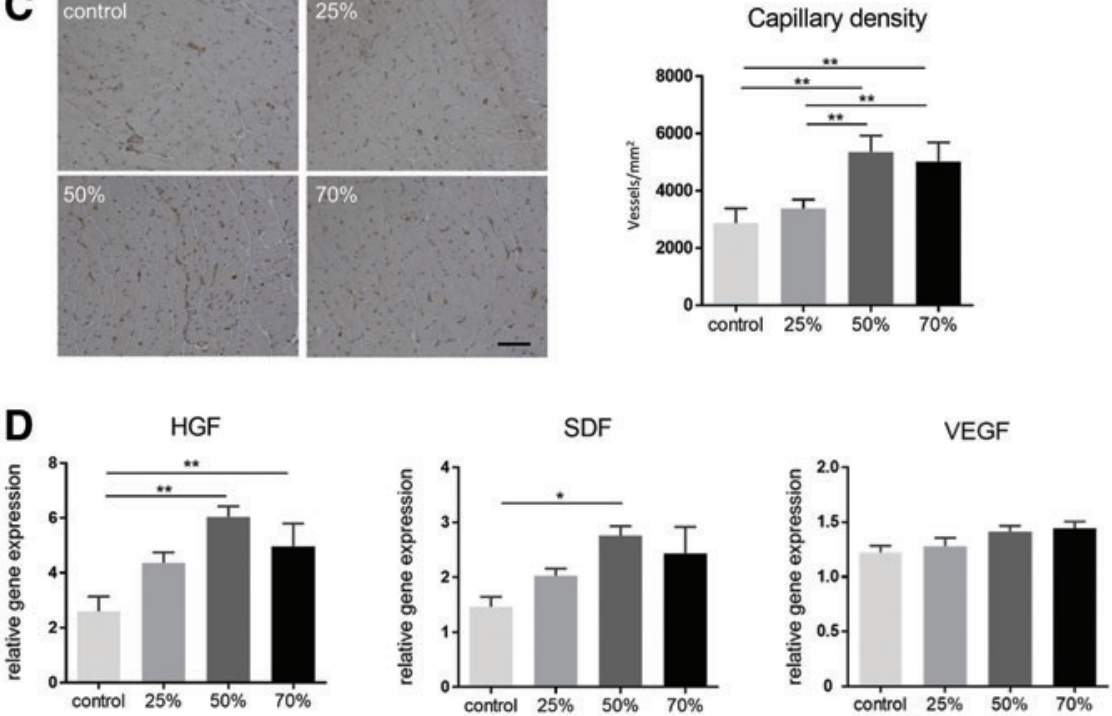

E
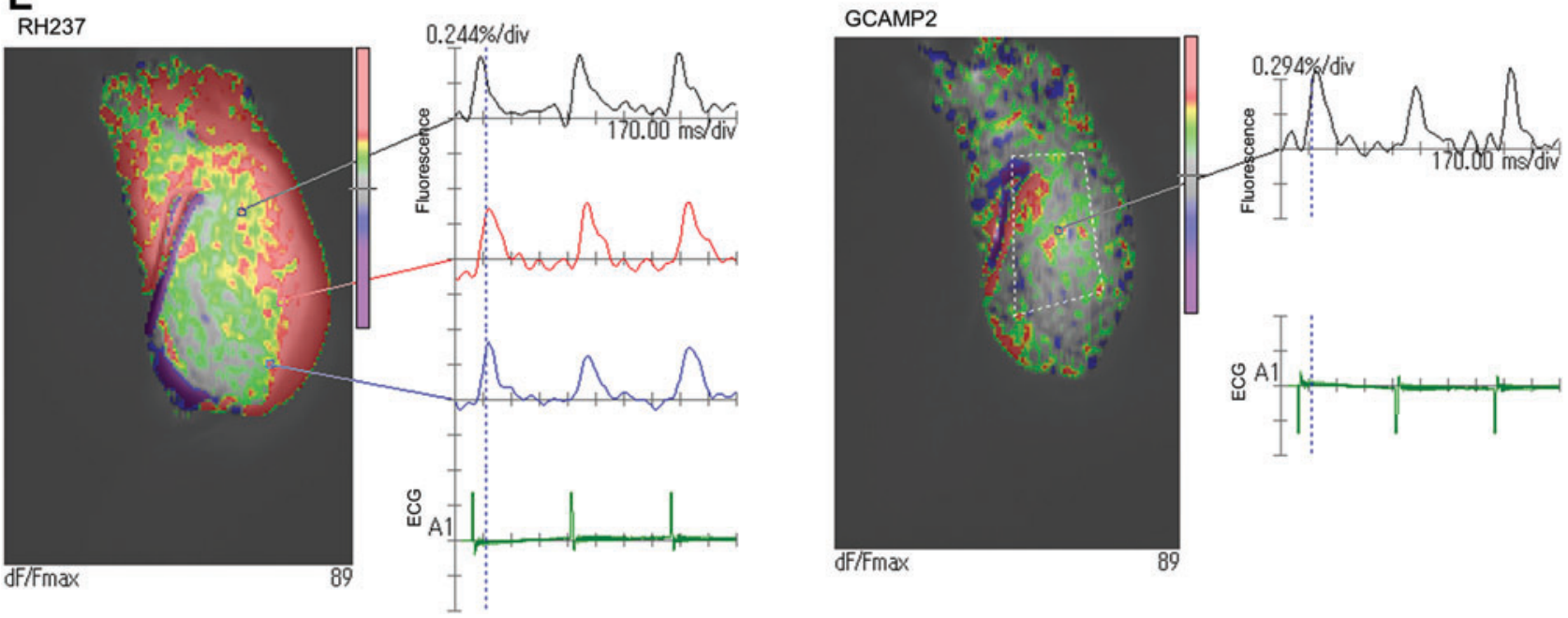

FIG. 8. Therapeutic potential of ECTs in a rat model of MI. Myocardial fibrosis (A, B) and capillary density (C) in the peri-infarct area 4 weeks after ECT transplantation was assessed by using Sirius Red staining and immunohistochemistry, respectively. (D) $H G F, S D F-1$, and $V E G F$ expression in the peri-infarct area of the native myocardium at 4 weeks after ECT transplantation was assessed by real-time PCR. Electrical integrity of the grafted ECT in the native heart was assessed in Langendorff-perfused hearts from rats transplanted with GCAMP2-expressing ECTs. (E) Electrical coupling between the native heart and transplanted ECT (dashed line) was analyzed by detection of $\mathrm{Ca}^{2+}$ transient (GCAMP2) and membrane potential (RH-237). Representative images of hearts transplanted with ECTs containing 70\% cardiomyocytes. Scale bars: $50 \mu \mathrm{m} . * p<0.05 ; * * p<0.01$. HGF, hepatocyte growth factor. Color images available online at www.liebertpub.com/tea 
characteristics that are associated with the extracellular environment. Another possible role of non-cardiomyocytes is production of trophic factors such as cytokine or miRNA. The content of non-cardiomyocytes affected the expression pattern of cytokines, as indicated in Figure 6E. It is reported that non-cardiomyocytes influence differentiation, maturation, or function of cardiomyocytes through trophic factors. ${ }^{18}$ In contrast, in vitro expression of the gap-junction protein connexin 43 did not differ substantially between groups, indicating that further studies are warranted to explore factors that determine the expression of these proteins to enhance ECT functionality and consistency.

For clinical application of iPSC-derived cardiomyocytes, phenotypic heterogeneity of ECT cardiomyocytes-such as ventricular or atrial subtype-remains an unresolved issue. ${ }^{19-21}$ This study explored the expression patterns of structural proteins such as MLC to assess the maturity of the cardiomyocytes populating the ECTs. Given that immature cardiomyocytes express both MLC2a and MLC2v, ${ }^{22}$ a higher ratio of MLC2 $\mathrm{v}$ single-positive cardiomyocytes in the ECTs containing $50 \%$ or $70 \%$ cardiomyocytes indicated a more mature ventricular phenotype, thus explaining their enhanced therapeutic potential in chronic MI rat hearts. Moreover, the therapeutic potential of ECT grafts increased depending on cardiomyocyte content. Importantly, electrical coupling between the native heart and grafted ECT was associated with therapeutic benefit, indicating that the mechanical and paracrine effects associated with HGF expression positively contributed to the overall effect. However, clear gapjunction formation between native and grafted cardiomyocytes was not identified by immunohistological analysis. Thus, intravital microscopy studies might be warranted to prove direct communication between the native tissue and grafted cells.

The absence of well-established vascular networks in vitro may limit the functionality of these results, as well as the assessment of future potential applications of ECTs. Vascular networks are critical not only in oxygen/nutrition delivery and waste excretion but also in providing structural stability to cardiac tissue. The addition of a suitable in vitro vascular network would enhance functionality and consistency of the ECT, enabling generation of larger grafts and enhancing their functional potential.

In conclusion, our results showed that the quantity of noncardiomyocyte stromal cells is critical in generating functional human iPSC-derived, scaffold-free ECTs for cardiac regeneration therapy by ECM production. Most significantly, we found that ECTs containing 50-70\% cardiomyocytes formed stable structures, produced enhanced electrical and mechanical functions, thereby increasing the overall cardiotherapeutic potential.

\section{Acknowledgments}

The authors thank Professor Junichi Nakai for kindly providing the GCAMP2 gene. They also thank Atsuko Wakimura, Hiromi Nishinaka, Tsuyoshi Ishikawa, and Nobu Miyakawa for expert technical assistance. In addition, they appreciate the invaluable advice provided by Dr. Akira Yoshida, Dr. Teruyuki Yokoyama, and Dr. Keiko Miwa. This work was supported by the Japan Agency for Medical Research and Development as part of the Research Project for Practical Application of Regenerative Medicine.

\section{Disclosure Statement}

No competing financial interests exist.

\section{References}

1. Lalit, P.A., Hei, D.J., Raval, A.N., and Kamp, T.J. Induced pluripotent stem cells for post-myocardial infarction repair: remarkable opportunities and challenges. Circ Res 114, 1328, 2014.

2. Matsa, E., Sallam, K., and Wu, J.C. Cardiac stem cell biology: glimpse of the past, present, and future. Circ Res 114, $21,2014$.

3. Williams, A.R., Trachtenberg, B., Velazquez, D.L., McNiece, I., Altman, P., Rouy, D., Mendizabal, A.M., Pattany, P.M., Lopera, G.A., Fishman, J., Zambrano, J.P., Heldman, A.W., and Hare, J.M. Intramyocardial stem cell injection in patients with ischemic cardiomyopathy: functional recovery and reverse remodeling. Circ Res 108, 792, 2011.

4. Leobon, B., Garcin, I., Menasche, P., Vilquin, J.T., Audinat, E., and Charpak, S. Myoblasts transplanted into rat infarcted myocardium are functionally isolated from their host. Proc Natl Acad Sci U S A 100, 7808, 2003.

5. Miki, K., Uenaka, H., Saito, A., Miyagawa, S., Sakaguchi, T., Higuchi, T., Shimizu, T., Okano, T., Yamanaka, S., and Sawa, Y. Bioengineered myocardium derived from induced pluripotent stem cells improves cardiac function and attenuates cardiac remodeling following chronic myocardial infarction in rats. Stem Cells Transl Med 1, 430, 2012.

6. Kawamura, M., Miyagawa, S., Miki, K., Saito, A., Fukushima, S., Higuchi, T., Kawamura, T., Kuratani, T., Daimon, T., Shimizu, T., Okano, T., and Sawa, Y. Feasibility, safety, and therapeutic efficacy of human induced pluripotent stem cell-derived cardiomyocyte sheets in a porcine ischemic cardiomyopathy model. Circulation 126, S29, 2012.

7. Sekine, H., Shimizu, T., Dobashi, I., Matsuura, K., Hagiwara, N., Takahashi, M., Kobayashi, E., Yamato, M., and Okano, T. Cardiac cell sheet transplantation improves damaged heart function via superior cell survival in comparison with dissociated cell injection. Tissue Eng Part A 17, 2973, 2011.

8. Takebe, T., Enomura, M., Yoshizawa, E., Kimura, M., Koike, H., Ueno, Y., Matsuzaki, T., Yamazaki, T., Toyohara, T., Osafune, K., Nakauchi, H., Yoshikawa, H.Y., and Taniguchi, H. Vascularized and complex organ buds from diverse tissues via mesenchymal cell-driven condensation. Cell Stem Cell 16, 556, 2015.

9. Lu, H.F., Chua, K.N., Zhang, P.C., Lim, W.S., Ramakrishna, S., Leong, K.W., and Mao, H.Q. Threedimensional co-culture of rat hepatocyte spheroids and NIH/3T3 fibroblasts enhances hepatocyte functional maintenance. Acta Biomater 1, 399, 2005.

10. Matsuura, K., Masuda, S., Haraguchi, Y., et al. Creation of mouse embryonic stem cell-derived cardiac cell sheets. Biomaterials 32, 7355, 2011.

11. Matsuura, K., Masuda, S., Haraguchi, Y., Yasuda. N., Shimizu, T., Hagiwara, N., Zandstra, P.W., and Okano, T. Creation of human cardiac cell sheets using pluripotent stem cells. Biochem Biophys Res Commun 425, 321, 2012.

12. Dubois, N.C., Craft, A.M., Sharma, P., Elliott, D.A., Stanley, E.G., Elefanty, A.G., Gramolini, A., and Keller, G. sirPA is a specific cell-surface marker for isolating cardiomyocytes derived from human pluripotent stem cells. Nat Biotechnol 29, 1011, 2011. 
13. Kainuma, S., Miyagawa, S., Fukushima, S., Pearson, J., Chen, Y.C., Saito, A., Harada, A., Shiozaki, M., Iseoka, H., Watabe, T., Watabe, H., Horitsugi, G., Ishibashi, M., Ikeda, H., Tsuchimochi, H., Sonobe, T., Fujii, Y., Naito, H., Umetani, K., Shimizu, T., Okano, T., Kobayashi, E., Daimon, T., Ueno, T., Kuratani, T., Toda, K., Takakura, N., Hatazawa, J., Shirai, M., and Sawa, Y. Cell-sheet therapy with omentopexy promotes arteriogenesis and improves coronary circulation physiology in failing heart. Mol Ther 23, 374, 2015.

14. Beam, J., Botta, A., Ye, J., Soliman, H., Matier, B.J., Forrest, M., MacLeod, K.M., and Ghosh, S. Excess linoleic acid increases collagen I/III ratio and "stiffens" the heart muscle following high-fat diets. J Biol Chem 290, 23371, 2015.

15. Hayakawa, T., Kunihiro, T., Ando, T., Kobayashi, S., Matsui, E., Yada, H., Kanda, Y., Kurokawa, J., and Furukawa, T. Image-based evaluation of contraction-relaxation kinetics of human-induced pluripotent stem cell-derived cardiomyocytes: correlation and complementarity with extracellular electrophysiology. J Mol Cell Cardiol 77, 178, 2014.

16. Collier, P., Watson, C.J., van Es, M.H., Phelan, D., McGorrian, C., Tolan, M., Ledwidge, M.T., McDonald, K.M., and Baugh, J.A. Getting to the heart of cardiac remodeling; how collagen subtypes may contribute to phenotype. J Mol Cell Cardiol 52, 148, 2012.

17. Williams, C., Quinn, K.P., Georgakoudi, I., and Black, 3rd, L.D. Young developmental age cardiac extracellular matrix promotes the expansion of neonatal cardiomyocytes in vitro. Acta Biomater 10, 194, 2014.
18. Hsieh, P.C.H., Davis, M.E., Lisowski, L.K., and Lee, R.T. Endothelial-cardiomyocyte interactions in cardiac development and repair. Annu Rev Physiol 68, 51, 2006.

19. Synnergren, J., Améen, C., Jansson, A., and Sartipy, P. Global transcriptional profiling reveals similarities and differences between human stem cell-derived cardiomyocyte clusters and heart tissue. Physiol Genomics 44, 245, 2012.

20. Jung, G., and Bernstein, D. hiPSC modeling of inherited cardiomyopathies. Curr Treat Options Cardiovasc Med 16, $320,2014$.

21. Lee, Y.K., Ng, K.M., Lai, W.H., Chan, Y.C., Lau, Y.M., Lian, Q., Tse, H.F., and Siu, C.W. Calcium homeostasis in human induced pluripotent stem cell-derived cardiomyocytes. Stem Cell Rev 7, 976, 2011.

22. Yang, X., Pabon, L., and Murry, C.E. Engineering adolescence: maturation of human pluripotent stem cellderived cardiomyocytes. Circ Res 114, 511, 2014.

Address correspondence to: Yoshiki Sawa, MD, PhD

Department of Cardiovascular Surgery Osaka University Graduate School of Medicine Yamadaoka, 2-2, Suita-city Osaka 565-0087

Japan

E-mail: sawa-p@surg1.med.osaka-u.ac.jp

Received: December 4, 2016

Accepted: May 3, 2017

Online Publication Date: June 13, 2017 